\title{
Introducing the concept of the isodose for optimisation of decontamination activities in a radioactive fallout scenario
}

Paper

Hinrichsen, Yvonne; Finck, R.; Rääf, C.; Andersson, Kasper Grann

Published in:

Journal of Radiological Protection

Link to article, DOI:

10.1088/1361-6498/aadd24

Publication date:

2018

Document Version

Peer reviewed version

Link back to DTU Orbit

Citation (APA):

Hinrichsen, Y., Finck, R., Rääf, C., \& Andersson, K. G. (2018). Introducing the concept of the isodose for optimisation of decontamination activities in a radioactive fallout scenario: Paper. Journal of Radiological Protection, 38(4), 1293-1310. https://doi.org/10.1088/1361-6498/aadd24

\section{General rights}

Copyright and moral rights for the publications made accessible in the public portal are retained by the authors and/or other copyright owners and it is a condition of accessing publications that users recognise and abide by the legal requirements associated with these rights.

- Users may download and print one copy of any publication from the public portal for the purpose of private study or research.

- You may not further distribute the material or use it for any profit-making activity or commercial gain

- You may freely distribute the URL identifying the publication in the public portal 


\title{
Introducing the concept of the isodose for optimization of decontamination activities in a radioactive fallout scenario
}

\author{
Y Hinrichsen ${ }^{1}$, R Finck $^{2}$, C Rääf ${ }^{2}$, K G Andersson ${ }^{1}$ \\ 1 Technical University of Denmark, Center for Nuclear Technologies, \\ Frederiksborgvej 399, 4000 Roskilde, Denmark \\ ${ }^{2}$ Lund University, Department of Translational Medicine, Medical Radiation \\ Physics, SUS Malmö, 20502 Malmö, Sweden \\ E-mail: yvhi@dtu.dk
}

\begin{abstract}
.
In the recovery phase after a radioactive release incident, it is important to be able to focus decontamination operations on the areas that contribute most to the radiation dose. Monte Carlo simulations were applied to determine the shielding effect of a building against radiation from various directions, also giving information on the dose contributions at various locations inside the building from specific areas outside. The concept of the isodose was developed to optimize decontamination activities, and was applied as isodose lines to define the smallest areas that lead to a certain dose reduction through decontamination of areas surrounding the building. The shape and position of the isodose lines depend on the geometry of the building, the wall thickness and material, and the observation point inside the building. Calculations have been made with a surface resolution of $1 \mathrm{~m}^{2}$ for four observation points in a modular building, assuming depositions of ${ }^{137} \mathrm{Cs}$ and ${ }^{60} \mathrm{Co}$ on the ground surface and on the roof, as well as $1 \mathrm{~cm}$ below the ground surface to represent ground penetration. For example, a ten times as larger area would have to be decontaminated to increase the dose reduction from 10 to $30 \%$, if it is assumed that all the contamination is located at a depth of 1 $\mathrm{cm}$.
\end{abstract}

Submitted to: J. Radiol. Prot. 


\section{Introduction}

After an airborne release of radionuclides to a populated area, external gamma irradiation from deposited radioactive material can contribute considerably to the radiation exposure of the inhabitants. The shielding of gamma radiation by buildings can, however, reduce this exposure, but as buildings generally have complex geometries their shielding properties will vary depending on where the radionuclides are deposited, and in which parts of the building the residents spend most of their time. Thorough and consistent removal of a topsoil layer can remove more than $90 \%$ of the contamination from the surface (Andersson 2009a), but equipment, consumables and skilled personnel are required, and the resulting costs can be high depending on the area to be treated (Roed et al. 2006). To minimize these costs while achieving a satisfactory dose reduction, it is useful to have information on exactly which areas it would be most beneficial to decontaminate, taking into account the shielding properties of local buildings.

As the geometry of buildings is too complex for simple methods such as the point kernel model, Monte Carlo calculations are needed to calculate their shielding properties (Jensen \& Thykier-Nielsen 1989). The applicability of the transport code MCNP6 (Goorley et al. 2012) in determining exposure reduction for a modular building type has been experimentally verified in a previous study by our group (Hinrichsen et al. 2018). The focus in that study laid on the comparison of experimentally determined and theoretically calculated shielding factors for ${ }^{137} \mathrm{Cs}$ and ${ }^{60} \mathrm{Co}$ sources that were positioned around and on top of a modular building, showing that an agreement within $2 \%$ can be obtained. As the next step, the aim of this study was to use the MCNP6 code to illustrate how the shielding properties of a modular building vary depending on the source location with respect to a number of different observation points (selected points where people could be located) inside the building.

Furthermore, the isodose concept for the optimization of decontamination activities is introduced. In its application as isodose lines for a 2-dimensional source area, for example, the ground or roof surface, it illustrates the extent to which the surrounding areas contribute to the external radiation exposure at the observation points inside a building. This could, in turn, be used to show how the decontamination of surfaces or replacement of topsoil can be optimized in an emergency situation by determining the surfaces that contribute most to the external radiation exposure at the various observation points, taking ground penetration into consideration, which is especially relevant for wet deposition scenarios involving, for example, nuclear power plant releases and nuclear weapons fallout.

\section{Materials and methods}

\subsection{Concept of the shielding factor}

Buildings naturally provide some shielding against radiation from the ground and from miscellaneous contamination of all outdoor surfaces (including soil, roofs, walls, windows and pavements), vegetation, and in the air (primary contaminant plume or resuspended radioactive matter). In the case of wet deposition of nuclear fallout, the 
contamination on the ground can generally be expected to contribute most to the total dose integrated over long periods of time (assuming that areas of soil in the local inhabited environment are not insignificant) (Andersson 2009b). Several different, and sometimes confusing, terms have been used to describe the shielding effect of a building, such as the shielding factor, reduction factor or protection factor. The term shielding factor is used here to describe the reduction of the absorbed dose rate inside the building compared to the absorbed dose rate outside the building, and represents the reduction of the absorbed dose rate by attenuation and scattering as the radiation passes through matter. Absorbed dose here refers to the absorbed dose free in air.

The shielding factor, $S_{b l d}$, at a point inside a building acting on a radiation source outside the building is here defined as:

$$
S_{b l d}=\frac{\dot{D}_{b l d}}{\dot{D}_{\text {ref }}}, \quad 0 \leq S_{b l d} \leq 1
$$

where $\dot{D}_{b l d}$ is the absorbed dose rate at a point inside the building and $\dot{D}_{\text {ref }}$ is the absorbed dose rate that would be measured at the same point if the building were removed after the radionuclide deposition. This factor is based on the barrier shielding factor concept, which was originally defined by Spencer (1962), and compares the dose rate at a given position resulting from the same source, to the dose rate at the same position when the building is replaced with air. Barrier shielding depends on the energy of the gamma radiation and on the composition and thickness of the absorbing material. Barrier shielding will also depend on the direction of the incident radiation on the building, as the attenuation will be higher when radiation is incident at oblique angles. Thus, barrier shielding varies with the angular direction of the photon fluence, and depends on the actual geometry of the source. The angular barrier shielding factor is therefore a function in two dimensions, the vertical and the horizontal.

For the sake of completeness it should be mentioned that a second concept was developed by Spencer (1962), called geometry shielding, which compares the dose rate at one position resulting from a given source when the building is replaced by air, to the dose rate above an infinite, uniformly contaminated plane surface source at a reference height of $1 \mathrm{~m}$. Geometry shielding can be combined with the barrier shielding concept by multiplication. The resulting factor is amongst others called the reduction factor.

The focus of this study is on barrier shielding and the angular contribution to the absorbed dose from ${ }^{137} \mathrm{Cs}$ and ${ }^{60} \mathrm{Co}$ sources on the surrounding ground, and on top of the same modular building (Figure 1) as used in our previous study (Hinrichsen et al. 2018). The term "shielding factor" is used in the following sections, as this study is concerned only with barrier shielding.

\subsection{Introducing the concept of the isodose}

Examination of the distribution of angular shielding factors around building structures leads to the conclusion that some contaminated areas have a higher influence on 
the radiation exposure inside the building than others. Considering that the distance between a given observation point inside the building and a given surface source element outside the building also influences the radiation exposure at the observation point, it is interesting to study which contaminated areas have the highest impact on the radiation exposure indoors. This could be helpful in optimizing decontamination activities after the release of radioactive substances and reducing the resulting waste.

In this study, an observation point $i$ is defined such that it can be inside or outside the building, and its position is described by the vector $\vec{r}_{i}$ in 3-dimensional space. The absorbed dose resulting from gamma-emitting radionuclides over an infinitesimal volume, $d V$, located at the positions $\vec{r}$, can be determined at this point. The dose contribution from $d V$ to the observation point $i$, here denoted the dose contribution density $\rho_{D, i}(\vec{r})$, can be determined at each point in space by, for example, defining a certain radionuclide contamination on a surface, or by defining it as 0 inside the building. Thus, the total dose $D_{i, \infty}$ at observation point $i$ can be determined by the following integral:

$$
D_{i, \infty}=\int \rho_{D, i}(\vec{r}) d V I F \lim _{\left|\vec{r}_{i}-\vec{r}\right| \rightarrow \infty} \rho_{D, i}(\vec{r})=0
$$

To determine the areas with the highest dose contribution, the concept of the "isodose" was developed in this study, as defined below.

Definition: The isodose $I D_{i, k}$ is defined by the outer boundary of one or more zones in space that contribute, for the most part, a given fraction $k$ to the dose at the observation point $i$. In the case when $\rho_{D, i}(\vec{r})$ is a continuous function with the maximum $\rho_{D, i, \max }<\infty$, the isodose $I D_{i, k}$ can be chosen from the range $0<I D_{i, k}<\rho_{D, i, \max }$ and the fraction of dose contribution $k_{i}$ resulting from the zone or zones determined by the isodose is given by:

$$
k_{i}=\int f\left(\rho_{D, i}(\vec{r})\right) d V / D_{i, \infty} \quad F O R \quad f\left(\rho_{D, i}(\vec{r})\right)= \begin{cases}\rho_{D, i}(\vec{r}), & \rho_{D, i}(\vec{r}) \geq I D_{i, k} \\ 0, & \rho_{D, i}(\vec{r})<I D_{i, k}\end{cases}
$$

In the case when $\rho_{D, i}(\vec{r})$ is not a continuous function for the entire space, for example, due to limitations of the considered space, the concept of the isodose is still applicable for jump discontinuities. In such a case, the left- and right-hand limits exist, and the respective point can be applied over the entire range of dose contribution densities that lie within the left- and right-hand limits.

These equations apply to a single observation point inside the building, but in a real fallout situation, decontamination must be performed in such way that a substantial dose reduction is achieved in an entire building. To obtain a measure of the effective shielding obtained in a building by a given decontamination measure, the times spent by the residents in various parts of the building must be accounted for. Therefore, so-called occupancy factors $p_{i}$ for various observation points can be applied to Equation (3) resulting in: 


$$
\begin{aligned}
& k=\int f\left(\rho_{D}(\vec{r})\right) d V / \sum_{i} D_{i, \infty} \cdot p_{i} \quad \forall \quad 1=\sum_{i} p_{i} \\
& \text { FOR } \quad f\left(\rho_{D}(\vec{r})\right)= \begin{cases}\sum_{i} \rho_{D, i}(\vec{r}) \cdot p_{i}, & \sum_{i} \rho_{D, i}(\vec{r}) \cdot p_{i} \geq I D_{k} \\
0, & \sum_{i} \rho_{D, i}(\vec{r}) \cdot p_{i}<I D_{k}\end{cases}
\end{aligned}
$$

Based on this definition, the concept of the isodose can also be applied to 2dimensional dose contribution densities $\rho_{D, i}(\vec{r})$, for example, on specific surfaces or on the ground surrounding the building. This can be done in some cases for the sake of simplification, when the depth distribution under a surface (e.g. fallout in the ground) is neglected.

\subsection{Applying the concept of the isodose}

In reality, we do not know the dose contribution density of the space (Equations (2) to (4)), and thus the concept must be applied for different scenarios. The first scenario could be a contaminated ground surface around a building. The depth distribution is neglected for the sake of simplification as it differs from point to point in a real fallout situation (see e.g., Östlund et al. 2017). Therefore, this application of the isodose concept is 2-dimensional, and the areas giving the highest dose contribution for a given sourcebuilding geometry can be determined using the following step-by-step procedure. To provide a better understanding, the relevant quantities are listed in Table 1.

Step 1: The dose resulting from the entire contaminated surface at the observation point $i, D_{i, \infty}$, is determined, as well as the contribution to the dose from smaller parts of the surface $D_{i, j}$. The size of the areas $A_{j}$ is chosen by reasonability based on the applied scenario. The dose contribution density is then calculated for each subarea using the relation $\rho_{D, i, j}=D_{i, j} / A_{j}$. If more than one observation point is considered, this process must be repeated for all points, and the results weighted with their respective occupancy factors $p_{i}: D_{j}=\sum_{i} D_{i} \cdot p_{i}$ for the dose contribution and by $\rho_{D, j}=\sum_{i} D_{i, j} \cdot p_{i} / A_{j}$ for the dose contribution density.

Step 2: The subarea $\mathrm{j}$ with the highest dose contribution density for the observation point $\rho_{D, i, j}$ or weighted dose contribution density $\rho_{D, j}$ is then determined.

Step 3: The fraction of dose contribution $k_{i}$ is then calculated by dividing the dose contribution $D_{i, j}$ by the dose resulting from the entire contaminated surface $D_{i, \infty}$ for one observation point, or for the weighted fraction of dose contribution $k$ by dividing $D_{i, j}$ by $D_{\infty}=\sum_{i} D_{i, \infty} \cdot p_{i}$.

Step 4: This subarea $j$ now represents the area with a fraction of dose contribution $k_{i}$ or $k$ surrounded by the isodose criterion $I D_{i, k}$ and $I D_{k} . I D_{i, k}$ and $I D_{k}$ are in turn defined here by their respective dose contribution densities $\rho_{D, i, j}$ or $\rho_{D, j}$.

Step 5: The subarea $\mathrm{j}$ with the next highest dose contribution density for the observation point $\rho_{D, i, j}$ or weighted dose contribution density $\rho_{D, j}$ is then determined. 
Table 1. Overview of the relevant quantities in the determination of the isodose.

\begin{tabular}{ll} 
Symbol & Name \\
\hline$A_{j}$ & Size of subarea $j$ \\
\hline$D_{\infty}$ & Total dose weighted for several observation points \\
$D_{i}$ & Dose at observation point $i$ \\
$D_{i, j}$ & Dose at observation point $i$ resulting from contamination of subarea $j$ \\
$D_{i, \infty}$ & Total dose at observation point $i$ \\
$D_{j}$ & Dose weighted for several observation points resulting from contamina- \\
& tion of subarea $j$ \\
\hline$i$ & Observation point index \\
$j$ & Subarea index \\
\hline$I D D_{i, k}$ & Isodose for the fraction of dose contribution $k$ to the observation point $i$ \\
$I D D_{k}$ & Isodose for the fraction of dose contribution $k$ weighted for several \\
\hline$k$ & observation points \\
$k_{i}$ & Fraction of dose contribution weighted for several observation points \\
\hline$p_{i}$ & Fraction of dose contribution to the observation point $i$ \\
\hline$\vec{r}$ & Occupancy factors \\
$\vec{r}_{i}$ & Any point in the defined space \\
\hline$\rho_{D}$ & Position of the observation point $i$ \\
$\rho_{D, i}$ & Dose contribution density weighted for several observation points \\
$\rho_{D, i, j}$ & Dose contribution density to the observation point $i$ \\
$\rho_{D, j}$ & Dose contribution density for the observation point $i$ resulting from \\
& Contamination of subarea $j$ \\
& Dosulting from contamination of subarea $j$ \\
\end{tabular}

Step 6: The fraction of dose contribution $k_{i}$ is calculated by dividing the sum of all dose contributions from the subareas determined in Step 2 or 5 so far, $\sum_{j} D_{i, j} \forall j: \rho_{D, i, j} \geq I D_{i, k}$, by the dose resulting from the entire contaminated surface $D_{i, \infty}$ for one observation point using the isodose $I D_{i, k}$ as the criterion equalling the dose contribution density $\rho_{D, i, j}$ of the subarea $j$ that was determined in Step 5 . This must be done for the weighted fraction of dose contribution $k$ by dividing the weighted sum of all dose contributions considered $\sum_{j} D_{j} \forall j: \rho_{D, j} \geq I D_{k}$ by the weighted dose resulting from the infinite contaminated surface $D_{\infty}$, again using the isodose $I D_{k}$ as the criterion equalling the dose contribution density $\rho_{D, j}$ of the subarea $j$ that was determined in Step 5.

Step 7: The subareas $j: \rho_{D, i, j} \geq I D_{i, k}$ or $j: \rho_{D, j} \geq I D_{k}$ that were determined in Step 2 or 5 now represent the area or areas with a fraction of dose contribution $k_{i}$ or $k$ surrounded by the isodose criterion $I D_{i, k}$ or $I D_{k}$ which are defined here by their respective dose contribution densities, $\rho_{D, i, j}$ or $\rho_{D, j}$ as in Step 6.

Step 8: Repeat Steps 4-7 until all subareas have been considered.

These Steps can be expressed more mathematically for one observation point by: 


$$
k_{i}=\sum_{j} D_{i, j} / D_{i, \infty} \quad \forall \quad j: \rho_{D, i, j} \geq I D_{i, k}
$$

and for more than one observation point by:

$$
k=\sum_{j} D_{j} / D_{\infty} \quad \forall \quad j: \rho_{D, j} \geq I D_{k}
$$

If the sizes of the areas $A_{j}$ are numerically equal, the procedure can be simplified as the determination of the dose contribution density for each subarea becomes unnecessary, and the dose contribution per subarea can be compared with the isodose $I D_{i, k}$ multiplied by the size of one subarea $A$. Mathematically, Equation (5) for one observation point can be transformed into:

$$
k_{i}=\sum_{j} D_{i, j} / D_{i, \infty} \quad \forall \quad j: D_{i, j} \geq I D_{i, k} \cdot A \quad \wedge \quad A_{j}=A
$$

and Equation (6) for more than one observation point into:

$$
k=\sum_{j} D_{j} / D_{\infty} \quad \forall \quad j: D_{j} \geq I D_{k} \cdot A \quad \wedge \quad A_{j}=A
$$

\subsection{Description of the calculations}

The Monte Carlo calculations for the modular building scenario were performed with the transport code MCNP6 (Goorley et al. 2012), using the cross-section data set ENDF/B-VII.0 (Chadwick et al. 2006). Among other processes, it accounts for photon creation and loss through relevant mechanisms such as bremsstrahlung, fluorescence, Compton scattering, photon capture, pair production and p-annihilation. The code allows for the definition of complex 3-dimensional geometries through a combinatorial geometry technique. The definition of the geometry is based on the construction drawings and descriptions made available by Bilsby (Bilsby ${ }^{\circledR}$ n.d.), and measurements at a real modular building, including an additional construction of breeze blocks that was used in our previous study (Hinrichsen et al. 2018) (Figure 1). The regions in space were constructed by a logical combination (union, intersection, difference) of elementary geometric bodies and surfaces. Data from various databases (McConn Jr et al. 2011, Websites 2016) were used to assign the material specifications with definite atomic compositions and densities as the input for the different building structures and environmental regions.

${ }^{137} \mathrm{Cs}$ sources with a primary gamma energy of $0.662 \mathrm{MeV}$, and ${ }^{60} \mathrm{Co}$ sources with primary gamma energies of $1.173 \mathrm{MeV}$ and $1.332 \mathrm{MeV}$, taking into account the emission probabilities of 0.9985 photons per disintegration for a primary gamma energy of $1.173 \mathrm{MeV}$ and 0.999826 photons per disintegration for a primary gamma energy of $1.332 \mathrm{MeV}$, were assumed. The source regions were defined as cylinders with a diameter and height of $1 \mathrm{~cm}$, on a $1 \mathrm{~m} \mathrm{x} 1 \mathrm{~m}$ grid, up to a lateral distance of $10 \mathrm{~m}$ from the sides of the modular building at the bottom of the modules and additionally, 




Figure 1. Birds-eye view of the model of the modular building.

at the top of the modules. For the assumption of homogeneous contamination, interpolation methods were applied to the results of the grid points. Internal scattering within the source material was neglected, and no (extra) material composition was assigned to the source regions. Separate Monte Carlo computations were performed for reference values without the building, in the same way as in the calculations when the building was present $\left(\dot{D}_{r e f}\right.$ in Equation (1)). Calculations with an infinite surface source on ground level, excluding the area of the modular building, were performed to determine the isodose lines. Furthermore, calculations were performed for surface sources of various radii centred in the centre of the modular building on the ground and $1 \mathrm{~cm}$ beneath it, to determine the impact of ground penetration.

The composition of the soil surrounding the building was based on data in a material compendium (McConn Jr et al. 2011). The soil in the model was defined as a $5 \mathrm{~m}$ deep slab, and the air above as a $500 \mathrm{~m}$ high slab, to take possible scattering effects into account. The observation points were defined as air-filled spheres with a diameter of $30 \mathrm{~cm}$, for which the absorbed dose free in air, was calculated. The four detector regions were centred at the corresponding observation points $1 \mathrm{~m}$ above the floor of the modules (Figure 2). Detector region No. 1 was centered at the observation point in the middle of the breeze block structure, No. 2 in the middle of one half of one module, No. 3 was $0.5 \mathrm{~m}$ away from a window, and No. 4 opposite a doorway and an opening between the modules. These locations were chosen as they represent different parts of the building, and are the same as in our previous study (Hinrichsen et al. 2018). The number and energies of the gamma particles passing through these regions were determined. The fluence was then transformed into dose using conversion coefficients (ICRP 2010). 




Figure 2. Observation points inside the modular building. The walls at each end of the building have two windows each. The left and right wall have one door each, and there is a opening connecting the two modules. The U-shape in the top-left corner represents the breeze block structure (LECA - Lightweight Expanded Clay Aggregate).

Several techniques can be applied to reduce the variation in the results below a standard deviation of $5 \%$ with acceptable computation times in MCNP6. The defined regions in space are called cells, and weight windows were generated for each cell. The number of a particle's weight in MCNP6 represents the number of physical particles, which in these calculations are photons, where different random walks are represented by one MCNP particle. A lower bound on the weight of particles in each cell is defined by the user, and the upper weight bound is a specified multiple of the lower bound. These weight bounds define a window of acceptable weights. If the weight of a particle emitted from the source and generated by interactions with the materials is below the lower weight bound, then the weight of the particle is randomly increased to a value within the window, or it is not included in the calculations. If the weight of a particle is above the upper limit, it is split so that its parts are within the window. No action is taken for particles with weights within the window. The weight windows were determined using the Weight Window Generator, which estimates the importance of the cells in space. The importance of cells is defined as the expected score generated by a unit weight particle after entering the cell. The average importance of each cell can be estimated using the cell-based generator. 


\section{Results and discussion}

\subsection{Shielding factors around a modular building}

In order to illustrate the influence of the position of the sources around the building on the shielding factor (Equation (1)), the results for ${ }^{137} \mathrm{Cs}$ and ${ }^{60} \mathrm{Co}$ are plotted in heat maps for all 4 observation points defined above (Figure 3 ). The shielding factor is substantially lower at observation point No. 1 (inside the breeze block construction) than at the other observation points. At this point, the overall average shielding factor is $0.29 \pm 0.20(1 \mathrm{SD})$ for ${ }^{137} \mathrm{Cs}$ and $0.37 \pm 0.21$ for ${ }^{60} \mathrm{Co}$. The shielding factors at the other points were about $0.72 \pm 0.22$ for ${ }^{137} \mathrm{Cs}$ and $0.77 \pm 0.21$ for ${ }^{60} \mathrm{Co}$.


Figure 3. Computed angular shielding factors according to source region position around a modular building, at four different observation points inside the building (indicated by the red dots) for ${ }^{137} \mathrm{Cs}$ and ${ }^{60} \mathrm{Co}$.

For ${ }^{137} \mathrm{Cs}$, the shielding factor of one module wall is $0.90 \pm 0.01$ and for one breeze 
block wall $0.25 \pm 0.01$; while the corresponding values for ${ }^{60} \mathrm{Co}$ are $0.94 \pm 0.01$ and $0.32 \pm 0.01$, respectively. As can be seen from the plots in Figure 3, even the doors and windows provide slightly better shielding than the module walls. The shielding factors determined for the doors and windows are $0.89 \pm 0.01$ and $0.85 \pm 0.01$ in case of ${ }^{137} \mathrm{Cs}$, and $0.93 \pm 0.01$ and $0.87 \pm 0.01$ in case of ${ }^{60} \mathrm{Co}$. All other shielding factors can be determined from combinations of these factors and the angles at which the radiation passes through the different materials. The impact of the angle of incidence at the observation point on the shielding factor depends on the increasing septum length of the material traversed by the gamma photons as the distance between the surface source element and the observation point decreases (close to the building). This effect is especially evident when the breeze block structure is in the line of sight between the source and the observation point. Hence, as the angle between the incoming radiation and wall approaches $90^{\circ}$, while moving further away from the observation point, the radiation penetrates less material, and thus the shielding factor is higher.

\subsection{Shielding factors on top of a modular building}

In order to demonstrate the influence on the shielding factor of different source positions on top of the building, the results for ${ }^{137} \mathrm{Cs}$ and ${ }^{60} \mathrm{Co}$ are plotted for the 4 observation points, as in case of the surrounding deposition (Figure 4). The shielding factor is substantially lower at observation point No. 1 (inside the breeze block construction) than for the other observation points. At this point, the overall average shielding factor is $0.32 \pm 0.30$ ( $1 \mathrm{SD}$ ) for ${ }^{137} \mathrm{Cs}$ and $0.38 \pm 0.29$ for ${ }^{60} \mathrm{Co}$. The shielding factors for the other points were about $0.59 \pm 0.22$ for ${ }^{137} \mathrm{Cs}$ and $0.66 \pm 0.19$ for ${ }^{60} \mathrm{Co}$.

All other shielding factors can be determined as combinations of radiation passing through the roof and then a module or brick wall, taking the angles at which the radiation passes through the different materials into account, as well as backscattering effects.

\subsection{Monte Carlo computed isodose lines around a modular building}

The concept of the isodose, in its simplified application for same-sized subareas, described in Equation (7), was applied to a case where decontamination would lead to different reductions in the absorbed dose, depending on which of the four observation points is being considered. Homogeneous ground contamination surrounding the modular building was assumed. The results are presented graphically as isodose lines in Figure 5 for ${ }^{137} \mathrm{Cs}$ and for ${ }^{60} \mathrm{Co}$.

It can be seen in Figure 5 that the shapes of the areas encompassed by the isodose lines for a given observation point are relatively similar for both radionuclides. The zones are larger for ${ }^{60} \mathrm{Co}$ as the source energy is higher than in the case of ${ }^{137} \mathrm{Cs}$. To give a better idea of the size of the area that would have to be decontaminated to achieve a certain reduction in relative dose, the respective values are presented in Table 2, together with the primary dose factor calculated for an infinite contaminated ground surface. The primary dose factor is directly related to the dose to the residents when no decontamination measures are taken and is given in pGy per $\gamma / \mathrm{mm}^{2}$ repre- 





Figure 4. Computed angular shielding factors according to source region position on top of a modular building at four different observation points inside the building (black dots) for ${ }^{137} \mathrm{Cs}$ and ${ }^{60} \mathrm{Co}$.

senting the dose (pGy) that would be caused by a homogeneous ground contamination for an source strength of one gamma photon per unit area $\left(\gamma / \mathrm{mm}^{2}\right)$.

The values vary significantly depending on the observation point, especially regarding the influence of the breeze block structure or walls, which cause backscattering of the radiation. There also appears to be an inverse correlation between the primary dose factor at a given observation point attributed to an infinite contaminated ground and the size of the decontaminated surface required to reduce this dose by a certain fraction. The lower the unremediated dose, the larger the size of the area that must be decontaminated to achieve a certain percentage of dose reduction. 



Figure 5. Isodose lines around a modular building at four different observation points inside the building (red dots) for ${ }^{137} \mathrm{Cs}$ and ${ }^{60} \mathrm{Co}$. The shading indicates the fraction of dose contribution to the observation point including the areas that are surrounded by the respective one. When the outside line for a certain dose reduction reaches the limit of the calculation grid, its shape might differ for a larger calculation grid.

\subsection{Monte Carlo computed isodose lines on top of a modular building}

The concept of the isodose applied to a 2-dimensional area, as described by Equation (5), was also applied to the roof of the building to determine the areas that would have to be decontaminated to achieve different degrees of reduction of the absorbed dose at the four different observation points. The results are presented graphically as isodose lines in Figure 6 for ${ }^{137} \mathrm{Cs}$ and for ${ }^{60} \mathrm{Co}$.

It can be seen from the figure that most of the dose contribution from the roof is resulting from a small area over each observation point. As in the case of ground penetration, the isodose lines for each observation point have similar shapes for the 
Table 2. Area $\left(\mathrm{m}^{2}\right)$ that would hypothetically have to be decontaminated to achieve various degrees of dose reduction at the four observation points in case of ${ }^{137} \mathrm{Cs}$ and ${ }^{60} \mathrm{Co}$ contamination. All contamination is assumed to be on the soil surface.

*The values for $30 \%$ dose reduction at observation point No. 1 were determined using a limited calculation grid, and the results may differ with a larger calculation grid.

Observation Radionuclide Primary dose factor be- Dose reduction

\begin{tabular}{llllll} 
point & fore decontamination & & \\
& & $\left(\mathrm{pGy}\right.$ per $\left.\gamma / \mathrm{mm}^{2}\right)$ & $10 \%$ & $20 \%$ & $30 \%$ \\
\hline \multirow{2}{*}{1} & ${ }^{137} \mathrm{Cs}$ & 173 & 48 & 155 & $396^{*}$ \\
& ${ }^{60} \mathrm{Co}$ & 394 & 51 & 168 & $450^{*}$ \\
\hline \multirow{2}{*}{2} & ${ }^{137} \mathrm{Cs}$ & 446 & 23 & 88 & 251 \\
& ${ }^{60} \mathrm{Co}$ & 829 & 27 & 106 & 303 \\
\hline \multirow{2}{*}{3} & ${ }^{137} \mathrm{Cs}$ & 489 & 15 & 58 & 172 \\
& ${ }^{60} \mathrm{Co}$ & 908 & 18 & 73 & 221 \\
\hline \multirow{2}{*}{4} & ${ }^{137} \mathrm{Cs}$ & 425 & 30 & 117 & 292 \\
& ${ }^{60} \mathrm{Co}$ & 804 & 37 & 140 & 349 \\
\hline
\end{tabular}

different zones and for both simulated radionuclides. Also here, the zones for ${ }^{60} \mathrm{Co}$ are larger as the emitted photon energy is higher than for ${ }^{137} \mathrm{Cs}$.

\subsection{Isodose lines based on more than one observation point}

So far, four separate observation points representing different parts of the building have been used to determine isodose lines. In the next step, occupancy factors $\left(p_{i}\right.$ in Equation (8)) are applied to determine isodose lines assuming a contaminated ground surface in order to obtain isodose lines that are more representative of the doses in the building as a whole. As it was observed that a smaller area had to be decontaminated to achieve a given dose reduction in the case of a high unremediated absorbed dose $D_{i, \infty}$ at a given observation point, the occupancy factors were chosen in proportion to the primary dose factors $p_{i}=D_{i, \infty} / \sum_{i} D_{i, \infty}$. The results are presented graphically in Figure 7. The size of the area that must be decontaminated to achieve dose reductions of $10 \%, 20 \%$, and $30 \%$ are $39 \mathrm{~m}^{2}, 125 \mathrm{~m}^{2}$, and $297 \mathrm{~m}^{2}$ for ${ }^{137} \mathrm{Cs}$ and $45 \mathrm{~m}^{2}$, $145 \mathrm{~m}^{2}$, and $353 \mathrm{~m}^{2}$ for ${ }^{60} \mathrm{Co}$. For the modular building studied, the primary dose factor based on the occupancy factors was determined to be 423 pGy per $\gamma / \mathrm{mm}^{2}$ for ${ }^{137} \mathrm{Cs}$ and 788 pGy per $\gamma / \mathrm{mm}^{2}$ for ${ }^{60} \mathrm{Co}$. In comparison with the values in table 2 , the inverse relation between primary dose factor and area size is still valid.

No isodose lines were determined for the roof taking all observation points into account as the results for the single observation points showed that the main dose contribution is from a small area over the observation point.

\subsection{Impact of ground penetration}

In order to study the impact of ground penetration on the size of an area encompassed by a given fraction of dose reduction, the absorbed doses resulting from contaminated 

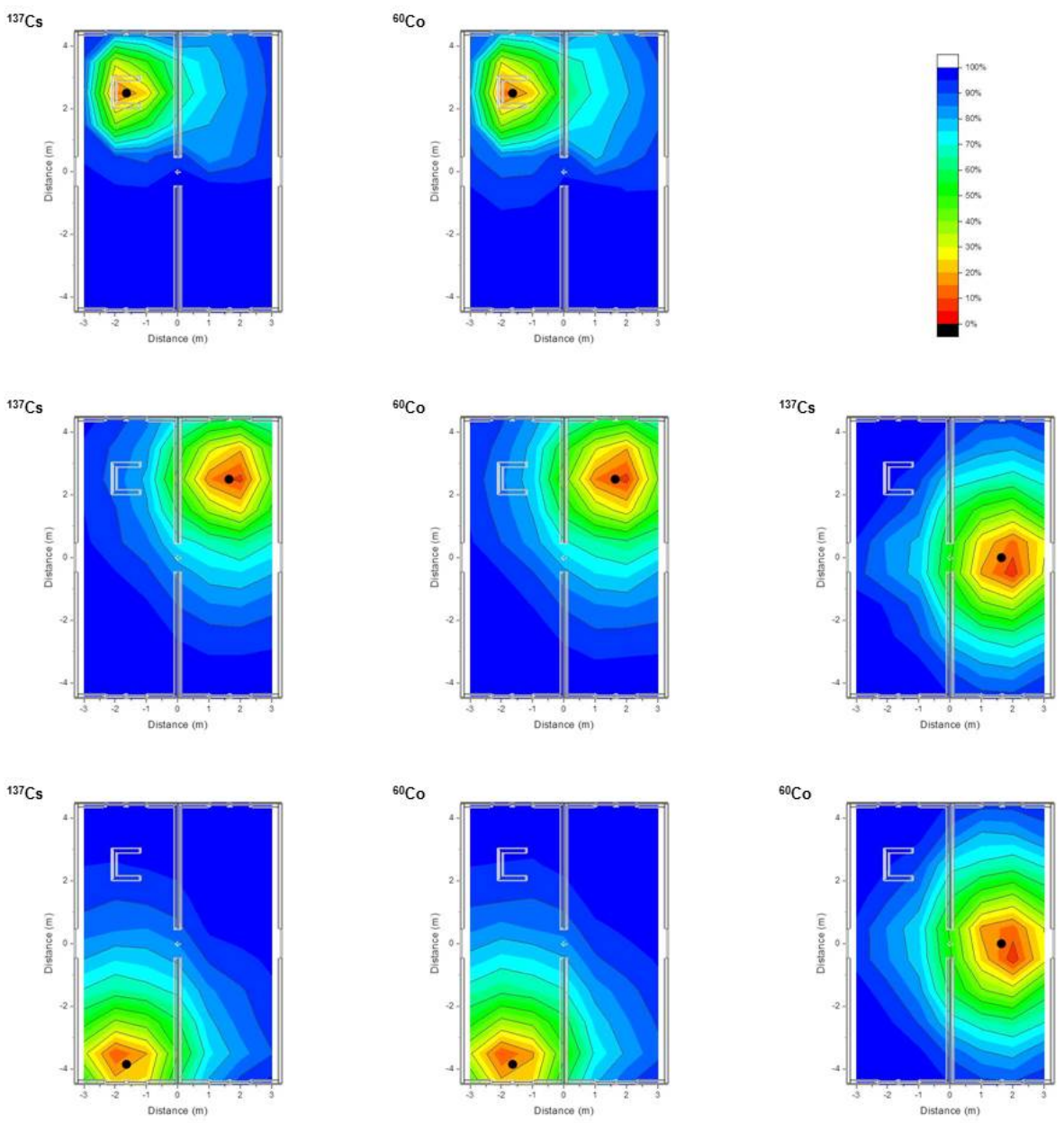

Figure 6. Isodose lines on top of a modular building at four different observation points inside the modules (black spot) for ${ }^{137} \mathrm{Cs}$ and ${ }^{60} \mathrm{Co}$. The areas of different colours represent the fraction of dose contribution to the observation point including the areas that are surrounded by the respective one.

circular areas of various sizes centred in the centre of the modular building on the ground were first calculated, and then divided by the absorbed dose resulting from an infinite ground source. The same calculations were then performed for a scenario with contamination to a depth of $1 \mathrm{~cm}$ beneath the ground surface, to simulate ground penetration of the fallout some time after the initial deposition event, as was the case for ${ }^{137} \mathrm{Cs}$ fallout from the Fukushima release (e.g., Östlund et al. 2017). Calculations were only performed for a ${ }^{137} \mathrm{Cs}$ source and the results are presented in Figure 8.

From Figure 8 it can be seen that decontaminating an average area of $1000 \mathrm{~m}^{2}$ would reduce the total absorbed dose contribution by about $45 \%$ without ground penetration, and by about $65-70 \%$ with ground penetration. To study the impact of ground penetration in more detail, the size of the circular area $A$ that would have to 



Figure 7. Isodose lines around a modular building for ${ }^{137} \mathrm{Cs}$ (left) and of ${ }^{60} \mathrm{Co}$ (right) using occupancy factors according to the primary dose for the four observation points. The areas of different colours represent the fraction of dose contribution including the areas that are surrounded by the respective one.



Figure 8. Size of a circular area that would have to be decontaminated to achieve a certain reduction in the total absorbed dose contribution at the four observation points (OP) inside the modular building. The continuous line indicates the average over all observation points without ground penetration, and the dashed line the average over all observation points assuming ground penetration.

be decontaminated to obtain a certain reduction in the dose contribution $f$ can be described by the mathematical expression:

$$
A(f)=\left(a \cdot(-\ln (1-f))^{b}+c\right)^{2}-59.4, \quad a, b, c>0
$$

Regression analysis can be used to obtain $\mathrm{a}, \mathrm{b}$ and $\mathrm{c}$ for each scenario, and the values are presented in Table 3 . The ground area of the modular building is $59.4 \mathrm{~m}^{2}$.

Comparing the values in Table 3 shows that a smaller area would have to be decontaminated in the case of ground penetration to obtain the same fractional dose reduction as in the case without ground penetration. Figure 9 shows the ratio of the 
Introducing the concept of the isodose

Table 3. Values of the variables in Equation (9)describing the fraction of absorbed dose contribution for areas of various sizes.

\begin{tabular}{lllll} 
Scenario & Observation point & Variable & \\
& & $\mathrm{a}$ & $\mathrm{b}$ & $\mathrm{c}$ \\
\hline \multirow{3}{*}{ Without ground penetration } & 1 & 68.6 & 1.72 & 9.57 \\
& 2 & 52.5 & 1.79 & 9.75 \\
& 3 & 44.4 & 1.82 & 9.57 \\
& 4 & 49.6 & 1.69 & 9.75 \\
\hline \multirow{3}{*}{ With 1 cm ground penetration } & 1 & 31.5 & 2.33 & 9.57 \\
& 2 & 21.3 & 2.50 & 9.75 \\
& 3 & 14.6 & 2.50 & 9.93 \\
& 4 & 22.9 & 2.44 & 9.57 \\
\hline
\end{tabular}

area that would have to be decontaminated without ground penetration to the area that would have to be decontaminated with ground penetration to obtain the same fractional dose reduction.



Figure 9. Ratio of the area that would have to be decontaminated without ground penetration to the area that would have to be decontaminated with 1 $\mathrm{cm}$ ground penetration to obtain the same fractional dose reduction at the four observation points.

The ratios in Figure 9 vary between 1 and 5.5, showing a peak at a dose reduction of in the range from $40 \%$ to $55 \%$. In the case of real fallout situations, it should be borne in mind that, according to the ICRP the key dose parameter to be considered in connection with decisions on interventions in a contaminated area is the residual dose, which is integrated over a long time for long-term interventions (ICRP 2007). Further factors are the costs and amounts of generated waste that are caused by respective countermeasures as well as the possible risk of erosion that could be increased by topsoil removal over large areas and the resulting social, environmental and ethical factors (Roed et al. 2006). Downward migration of the contamination in soil over time 
can lead to different fractional contributions to the dose from contamination at different distances, as can be seen in Figure 8. Therefore, to fully comply with the ICRP recommendations, in a "real" situation, each calculated dose contribution at a given depth should be multiplied by the predicted fraction of the contamination present at that depth at each time, and it should all be integrated over time to give a committed dose over a given time span (e.g. 70 years). Downward penetration will depend greatly on the soil type and status, weather conditions, human and other impact, as well as on the physiochemical characteristics of the contaminants. These factors were neglected in this illustration of the method for the sake of simplicity, but they should be taken into account in realistic cases.

\section{Conclusions}

This study has demonstrated the influence of various building materials and the angle of incidence of radiation on the shielding factor in a modular building, using the Monte Carlo model MCNP6. For example, the breeze block structure provides the most efficient shielding, with about 3 times lower shielding factor than the walls of the modules. Even the doors and windows exhibit slightly lower shielding factors than the walls. Furthermore, the concept of the isodose, defining an area with a given dose contribution to a defined observation point inside the building, was introduced. Such isodose lines can be used to illustrate the extent of surrounding areas that must be decontaminated to achieve a certain dose reduction, depending on the observation point. It was found that the shape of the surface encompassed by the isodose lines did not change with increasing gamma energy of the incident photons, but the area requiring decontamination to achieve the same percentage of dose reduction increased with higher gamma energies. An inverse correlation was found between the primary dose factor and the size of the area that has to be decontaminated to achieve a certain dose reduction. To optimize decontamination activities for a residential building living habits inside the building must be considered, and a combination of primary dose factors for a number of observation points must therefore be used, depending on the occupancy times of the residents. When assuming $1 \mathrm{~cm}$ deep ground penetration, a decrease in the area requiring decontamination, of up to $60 \%$, was found compared with surface deposition. This means that a substantially smaller area will have to be decontaminated to achieve a given reduction in dose after allowing time for the gradual migration of the deposition.

In conclusion, the isodose concept shows promise for comparison of the effects of decontaminating different surface areas, for different primary gamma energies, and different degrees of ground penetration. Further studies are required on the application

of these findings to other types of buildings, as well as the choice of a representative indoor observation point for such buildings, in order to develop this method into a practical and useful instrument for the optimization of countermeasures in cases of radioactive fallout in inhabited areas. 
Introducing the concept of the isodose

\section{Acknowledgements}

This study was supported by the Swedish Radiation Safety Authority (SSM) and by the Swedish Civil Contingencies Agency (MSB).

\section{References}

Andersson, K. G. (2009a), Chapter 8 countermeasures for reduction of dose in contaminated inhabited areas, in K. G. Andersson, ed., 'Airborne Radioactive Contamination in Inhabited Areas', Vol. 15 of Radioactivity in the Environment, Elsevier, pp. 217 - 258.

Andersson, K. G. (2009b), Chapter10 strategies for restoration of contaminated inhabited areas, in K. G. Andersson, ed., 'Airborne Radioactive Contamination in Inhabited Areas', Vol. 15 of Radioactivity in the Environment, Elsevier, pp. 297 - 326.

Bilsby ${ }^{\circledR}$ (n.d.), 'Snittegning af kontormoduler - stabelbare; konstruktionsbeskrivelse - kontormoduler - stabelbare (in danish)'.

Chadwick, M., Obložinský, P., Herman, M., Greene, N., McKnight, R., Smith, D., Young, P., MacFarlane, R., Hale, G., Frankle, S., Kahler, A., Kawano, T., Little, R., Madland, D., Moller, P., Mosteller, R., Page, P., Talou, P., Trellue, H., White, M., Wilson, W., Arcilla, R., Dunford, C., Mughabghab, S., Pritychenko, B., Rochman, D., Sonzogni, A., Lubitz, C., Trumbull, T., Weinman, J., Brown, D., Cullen, D., Heinrichs, D., McNabb, D., Derrien, H., Dunn, M., Larson, N., Leal, L., Carlson, A., Block, R., Briggs, J., Cheng, E., Huria, H., Zerkle, M., Kozier, K., Courcelle, A., Pronyaev, V. \& van der Marck, S. (2006), 'Endf/b-vii.0: Next generation evaluated nuclear data library for nuclear science and technology', Nuclear Data Sheets 107(12), 2931 - 3060. Evaluated Nuclear Data File ENDF/B-VII.0.

Goorley, T., James, M., Booth, T., Brown, F., Bull, J., Cox, L. J., Durkee, J., Elson, J., Fensin, M., Forster, R. A., Hendricks, J., Hughes, H. G., Johns, R., Kiedrowski, B., Martz, R., Mashnik, S., McKinney, G., Pelowitz, D., Prael, R., Sweezy, J., Waters, L., Wilcox, T. \& Zukaitis, T. (2012), 'Initial mcnp6 release overview', Nuclear Technology 180(3), 298-315.

Hinrichsen, Y., Finck, R., Östlund, K., Rääf, C. \& Andersson, K. G. (2018), 'Comparison of experimental and calculated shielding factors for modular buildings in a radioactive fallout scenario', Journal of Environmental Radioactivity 189, 146 - 155.

ICRP (2007), 'The 2007 recommendations of the international commission on radiological protection', Annals of the ICRP 37(2-7), 1 - 332. ICRP Publication 103.

ICRP (2010), 'Conversion coefficients for radiological protection quantities for external radiation exposures', Annals of the ICRP 40(2), 1 - 257. ICRP Publication 116.

Jensen, P. H. \& Thykier-Nielsen, S. (1989), 'Shielding factor calculation for plume radiation', Radiation Protection Programme. Progress Report 1988 pp. 1561-1565.

McConn Jr, R., Gesh, C., Pagh, R., Rucker, R. \& Williams III, R. (2011), 'Radiation portal monitor project - compendium of material composition data for radiation transport modeling. pacific northwest national laboratory. piet-43741-tm-963, pnnl-15870 rev. 1'.

Östlund, K., Samuelsson, C., Mattsson, S. \& Rääf, C. L. (2017), 'The influence of (cs)-c-134 on the cs-137 gamma-spectrometric peak-to-valley ratio and improvement of the peak-to-valley method by limiting the detector field of view', Applied Radiation and Isotopes 128, 249-255.

Roed, J., Andersson, K. G., Barkovsky, A., Fogh, C., Mishine, A., Ponamarjov, A. \& Ramzaev, V. (2006), 'Reduction of external dose in a wet-contaminated housing area in the bryansk region, russia', Journal of Environmental Radioactivity 85(2-3), 265-279.

Spencer, L. (1962), 'Structure shielding against fallout radiation from nuclear weapons', United States Bureau of Standards - Monograph National Bureau of Standards Monograph 42.

Websites (2016), '(in german)'.

URL: www.isola-mineralwolle.de;https://de.wikipedia.org/wiki/N\%C3\%A4hrstoff_(Pflanze); http://www.kaiservrees.de/downloads/Ra40gew5chte.pdf;https://de.wikipedia.org/wiki/Brettsperrholz;https://de.wikipedia.org/wiki/Spanplatte;http:// 